\title{
Reference and Inheritance of Yi Costume Elements in Modern Design
}

\author{
Lixia Wang \\ Panzhihua University \\ Panzhihua, China 617000
}

\begin{abstract}
Yi costume has a long brilliant history, and the clothes are divided into different types for the different regions. The profile, the pattern and the special crafts of $\mathrm{Yi}$ costume can be directly applied to the modern design or reorganization, and then it would make a new artistic appeal. And the design of clothing accessories and textiles are both fashionable with the cultural characteristics. It makes Yi dress inherited and retained.
\end{abstract}

\section{Keywords-Yi costume; costume culture; inheritance}

\section{INTRODUCTION}

As the sixth largest ethnic minority in China, Yi nationality has a wide range of clothes and a rich dress culture, which deserves our research, discussion and study. To continue the inheritance and development of ethnic traditional costumes, we should not only protect them, but also combine them with modern design concepts and advanced techniques so that more modern people can accept them. And this paper mainly describes it from innovative design and practical applications.

\section{INTERPRETATION OF Yi COSTUME CULTURE}

\section{A. Cultural Background and Dress Outline of the Costume} of Yi Nationality

Yi nationality has a long history \& is the sixth largest ethnic group in China, there is a record of the $\mathrm{Yi}$ in the Chinese early history, it's called as Xinanyi in Han history, and they called themselves as " $\mathrm{Ni}$ ", because in the ancient Chinese, the" pronounced "Ni" is recorded as "Yi", thus, it's record as "Yi" in most of the Han book. Due to the historical reasons, one art of the ancestors of Diqiang ethnic goes into the current Xiaoliang mountain, Wumeng mountain, Ailao mountain and Yungui plateau from the northwest of Ganqing area; Daliang/Xiaoliang mountain, the northeast of Yunnan, northwest of Guizhou still have some nomadic characteristics, and due to the contact with the local people increased, the Yi nationality located in western, south of Yunnan, and western Sichuan gradually transformed into agricultural economy, and in order to adopt to the different life style, it is roughly differentiated into Liang mountain type, Wumeng mountain type, Honghe type and southeast of Yunnan type.

There're many kinds of Yi clothing in Liang mountain, and their styles and patterns are unique and colorful. According to the incomplete statistic, there're more than 300 kinds of clothing, and thousands kinds of accessories and patterns. Although it isn't the same in everywhere, but men usually wear black narrow sleeve coat with right surplice and trousers with multi fold and wide trousers; in some areas, men wear trousers with small trousers, and in the middle of the head front store up a little tuft long hair cidaroid and tie a knot on the right clamp. Women more likely retain national characteristic, usually wind Baotou, and has a corset and belt. Women in some areas have the habit of wearing longuette. Men and Women will wear the Phi felt when they go out. Yi nationality prefer the black color, thus all the costumes, utensils and so on mainly take the black as the background, and be supplemented by red, yellow and other colors. Although the costumes vary slightly because of their living conditions, they are basically the same in style. Most of the women wear the right lapel embroidered jacket, the collar is separated from the body, and the adult women mostly wear a pleated skirt, the youth ladies sometimes like to wear the self-made woolen skirt; The waist is equipped with sandwich triangle Decal pouch, and drooping with arrow shaped multicolored streamers and gorgeous spicule tube. The men dress of $\mathrm{Yi}$ nationality is divided into underwear and outerwear, most the underwear is the front opening white shirt without collar, and outerwear is made of black and blue colors, no decorative patterns, the young men coat tight with narrow sleeves; the bottom are the trousers made of cloth made of blue and black color, weave the white strips into strips to make trousers belts.

$\mathrm{Yi}$ women's ingenuity, most of them began to learn embroidery in childhood with his mother, so Yi dress patterns is very various, the traditional patterns have plate long lines, words flow pattern, fire pattern, claw pattern etc., and the Yi nationality advocates totems, and the totems on the clothes are different from each other because of the different species in the area.

\section{B. Traditional Costume and Accessories}

Because the author is from Panzhihua University in Panxi area, so here only discuss the Yi costume in Liang mountain area, and according to the local dialect, Liang Mountain Yi nationality can be divided into 3 dialect areas of Yinuo, Suodi, and Shengzha.

1) Costume: Yi nationality people in Yinuo area speak the "Yinuo" dialect, and commonly known as the "big trousers" area, the men's bottom of a trouser leg is wide and 
big like a skirt, the coat is tight with narrow sleeves, and there're decorations at the cuff and placket, mainly include Zhaojue, Meigu, Leibo, Ganluo and counties like this. The Suodi area is different from the big trousers area, which is called as the "small trousers" area because of its small trousers leg, the costume of Butuo area of Yi nationality is completely protected, which is the silver town of $\mathrm{Yi}$ nationality, and can display the old and pure culture of $\mathrm{Yi}$ nationality costume. Shengzha is named as "middle trousers" in common, which mainly include counties like Yuexi, Yanyuan, Xide, Mianning, Muli and so on. And among of them, Yuexi has fruitful cultural background, and is the key traffic of the old way in Lingguan, it's the original place for Wenchang culture and place of the fire was planted on the long march of the Red Army.

2) Accessories: The accessories of Yi People's clothing mainly include three kinds - headgear, jewelry and accessories, of which triangle bag is a typical kind of clothing accessories. The triangular bag is a triangular pouch hung by the waist of the $\mathrm{Yi}$ women in Liang Mountain. It is used for small change, sewing needles or tobacco leaves, and has double functions of beauty and utility, and in general, is divided as 3 kinds of Zhaojue, Meigu and Puge. The Zhaojue triangle bag is mainly made of collage, the red base is matched with the black pattern and lock the embroidery with yellow line. The Meigu triangle bag take the blue color as its main embroidery, the embroidery on the black base cloth is locked by the blue line, Puge triangle bag mainly take the collage and mosaic process and the decoration is big and prominent.

\section{ThE INHERITANCE OF Yi NATIONALITY'S COSTUME ELEMENTS}

\section{A. The Outline and Structure of National Costume}

Silhouette changes can bring new initial visual impact, so profile is the most basic problem involved in designing and making process. There are two commonly used borrowing methods: direct application and scatter reconstruction. There are many special costumes in Yi People's clothing, such as hawk mat, cutting integrated overclothes with half sleeves, which is valuable to us for learning. In the modern costume design, it can not only borrow from the profile but also can borrow the inner structure of the national costume, adopt the structures like coordination \& unification, comparison, proportion, repeat, cover, flexure and spare parts and so on to create clothes that are more suited to the wearing way of modern people.

\section{B. Pattern Color of Yi Nationality}

National pattern with the wide material resources for getting pattern is the most splendid color in national costume. They are often used in modern design in many ways. Take its essence, make it more applicable to the aesthetic needs of modern clothing. The costume patterns of Yi nationality are very rich in patterns, the typical patterns like Vortex pattern, fire pattern, horn pattern, Guozhuang pattern, toast seal pattern and so on, which generally displayed in Disc embroidery or embroidered technology. E.g.: Bulky yarn dish embroidery - Adopt the 'Spinning' to spin tightly the double color cotton line or 'bulked yarn' line, and embroider them into a variety of two square continuous pattern, which is mainly used in Meigu and Leibo areas, and be used in the cuff $\&$ placket of the jackets of men and women. Inclined disc embroidery --A process in which cloth is cut diagonally into strips and sewn into cloth and embroidered on a garment, which is most used in costume in Liuhong and Yuexi area, the craft of the inclined disc embroidery in Yuexi area is worthy of the best of Liang mountain.

\section{The Application of Yi Nationality's Arts and Crafts}

Many kinds of manual techniques of the traditional costume of Yi nationality, which include embroidery, disc embroidery, knitting and quilting and so on. These kinds of traditional manual techniques are not the important components of costume of Yi nationality, but also is a source of inspiration for us to carry out modern fashion design. The typical traditional technique is embroidery, in addition, there are folding process, felting process and Vegetation Dyeing Process etc.. It is different from the method of ironing irons in modern clothing technology, Sprinkle \& fold the pleat , use the foot to press the pleat, the pleat made by this kind of method is lively and vitality. Rolling felt is one of the traditional techniques of Yi nationality, which is mainly used to Phi felt, felt coat and felt hat and so on, it is an important clothing used for resisting the wind and cold. Vegetation dyeing is also known as plant dyeing, the natural plant is used as the dye to dye the fabric. Yi nationality in Liang mountain has taken the "Kui" as the mainly dyeing goods in the early time, thus the dyeing method of Yi nationality of Liang mountain also called as Kuiran(after dyeing).

In the modern fashion design, it can be accomplished either by the material reconstruction or by the reference of the decoration technique.

\section{The CREATION DESIGN Of The Costume ElEMENTS OF YINATIONALITY}

\section{A. Direct Application}

The direct use of national patterns and colors is divided into two kinds: one is to use the whole form of national costume design directly in modern design. The traditional totems of $\mathrm{Yi}$ nationality have many patterns. They can change their traditional patterns and apply them to costume design to the original charm of dress. The other is that the partial form of national costume is directly used in the design.

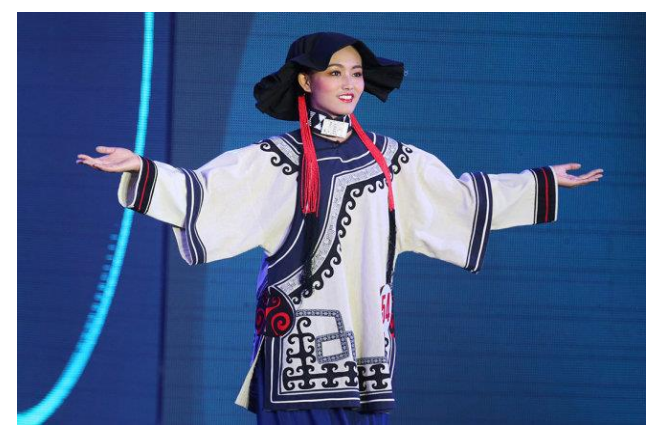

Fig. 1. The direct use of Yi costume' elements. 


\section{B. Scatter Reconstruction}

Scatter reconstruction is to sum up the styles and features of Yi People's clothing in the light of fully understanding the wearing methods and structure styles of ethnic costumes, change its common location and common decorative techniques and replace them with the adoption of patterns and colors imbued with the times. The common designing methods like the follows:

1) The association between meaning and meaning: Two or more than two things, although different in attributes, different in structure and different in shape, show the similar implication. The essence of meaning and meaning is not to rigidly adhere to the expression of form in design, but to pay attention to the understanding and interpretation of manner. E.g. When the clothes is being designed, adopt the ethereal Charm of the lotus hat of the Yi nationality for the whole design, cut the sleeves and the hem into the lotus shape to show the ethereal charm of the clothes.

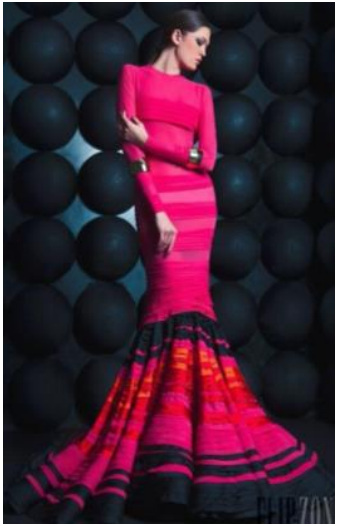

Fig. 2. The first sample of applications to refactoring Yi's elements.

2) The association between shape and shape: Two or more than two things in the shape or structure have similar morphology, which helps to cause the thought between the shape and things, and is good for creating a new form or structure and give it new meaning. It is possible that to adopt the style and structure of the traditional costume of Yi nationality, absorb the special patterns of it, simply the complex clothing style and then change them into the simple style for clothes that suitable for the modern people.

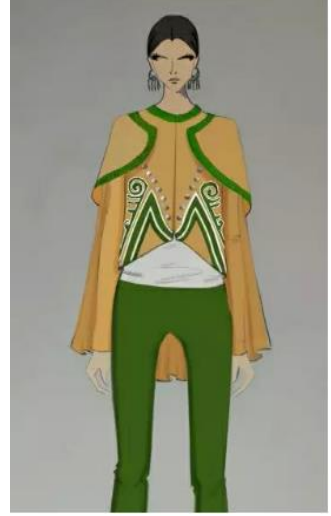

Fig. 3. The second sample of applications to refactoring Yi's elements.
3) The related thought: The related thought is a process of imaginative extension caused by the existence of related or necessary links between two or more than two things. E.g., learn the skirt processing method from the traditional pleated skirt, change the fold direction, and combine the tradition and the modern to make the modern clothing with great visual impact.

\section{APPLICATION OF Yi PEOPLE'S Clothing ELEMENTS IN TEXTILE PRODUCTS}

The costume of Yi nationality in Liang mountain area is the products of comprehensive factors, such as the specific history, human environment, natural environment; it is the integration of material and spiritual culture, which formed the dress style with complex structure, various patterns, decorative art workmanship, bright colors of Yi nationality in Liang mountain area. If one can deeply understand the deep culture connotation behind the culture of Yi nationality, know its natural beauty hiding in the mountain and then connect the traditional things with all aspects of our modern product, try to find the factors suitable for the modern society and create a new design. Here, I will present some research works on the application of $\mathrm{Yi}$ elements belonging to teachers and students of Panzhihua University

\section{A. Housewear \& Furnishings}

The patterns of the Yi people have unique beauty. We can not only use it in the clothing, but also apply it to the household decorations. For example, the curly grass pattern and disc embroidery technique on the pillow and lampshape in the left fig. has made the modern pillow has the unique national charm. It is not only the simple patterns for decoration, but also is the carrier of national culture.

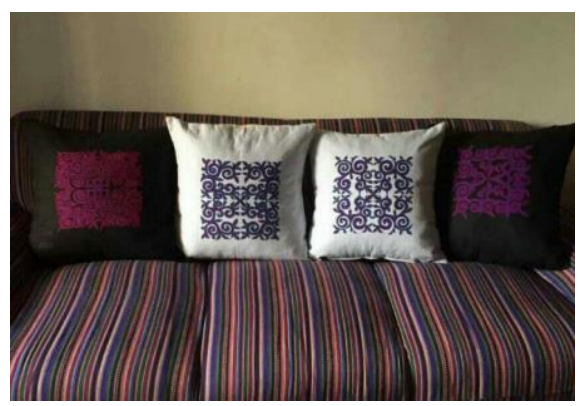

Fig. 4. One of the Yi patterns applied toHome Furnishing.

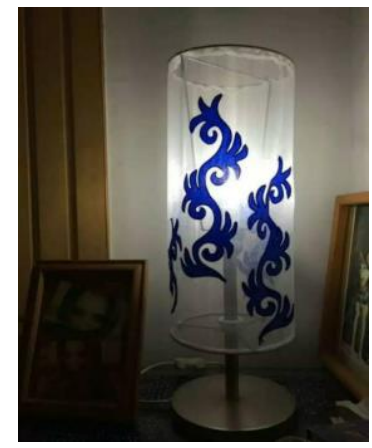

Fig. 5. One of the Yi patterns applied toHome Furnishing. 


\section{B. Accessories}

These accessories below are clothing works from the teachers and students of Panzhihua University; it combines the inclined disc embroidery and grape button knot combination process of Yuexi, and in the middle ring made by an inclined disc embroidery put the agate, crystal, beads made of obsidian, which create the ethnic style jewelry fully combining the tradition and fashion, and it reflect the aesthetic needs of people to return to the traditional generation.
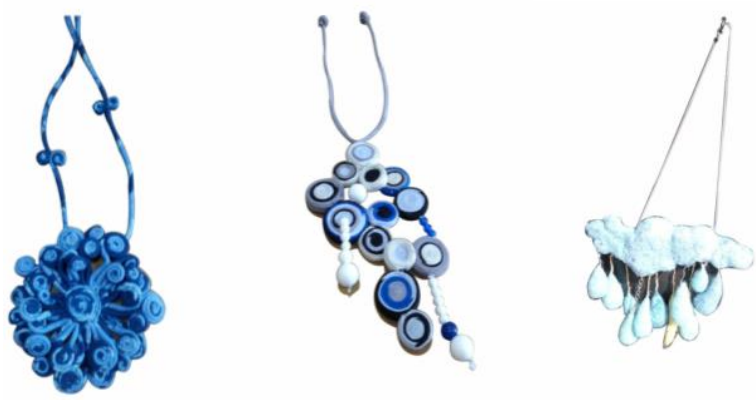

Fig. 6. The design of Yi accessories.

\section{Tea Culture Articles}

In the tea cultural articles, the special rolling felt process and the needle embroidery of the Yi nationality are used to make the materials of ordinary style into the tea table articles with unique style of the Yi people, which achieves the perfect integration of beauty and utility.

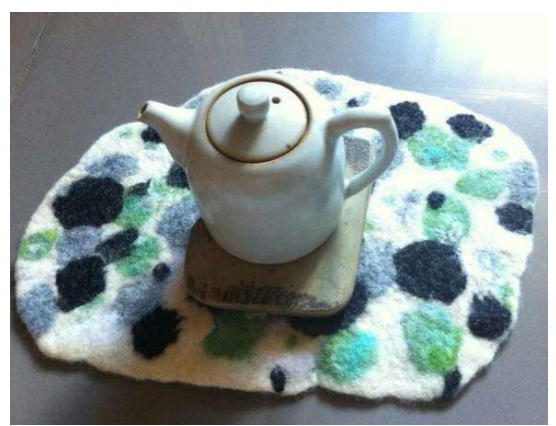

Fig. 7. Yi elements applied to the design of tea set.

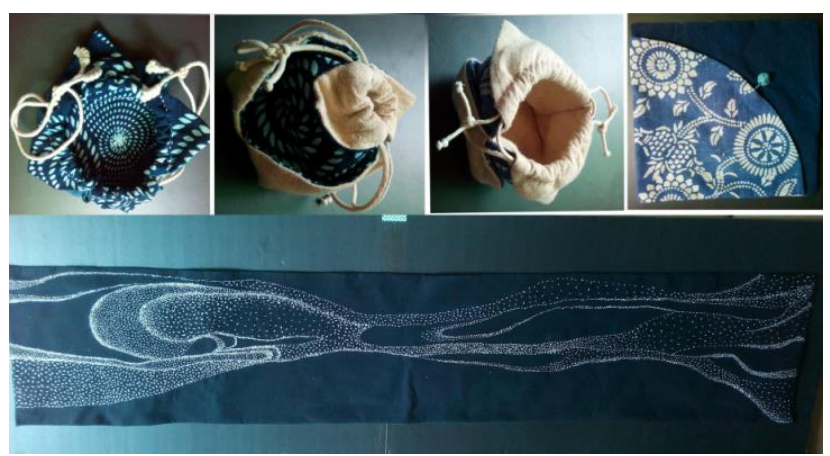

Fig. 8. Yi elements applied to the design of tea set.

\section{CONCLUSION}

In order to have the so-called international standards, many traditional things have been forgotten by us in the rapid development, once the traditional elements for many young designers are backward, rustic, and are discarded as the dross, so we found our dress seems more and more fashion, more and more "fan", but also more and more without Chinese "fan", more and more without our unique charm. Through the research and application of Yi dress in Liang mountain, find its mining points with the modern design elements and the individual elements contained by $\mathrm{Yi}$ dress itself in Liang mountain, which not only enrich the cultural products, but also increase the new visual light of modern textile including apparel, which make the Yi dress spirit of Liang mountain can be preserved in a new form in modern society.

\section{REFERENCES}

[1] Ye Julan Discussion on the application of the elements of Chuxiong Yi's costume design in modern design [J]. Art and Technology, 2016, 29 (4), P144-145

[2] You Lingli Study on the application of Yi nationality clothing elements of Liang mountain in modern fashion design [J]. 2013.12.20, master's thesis of Zhejiang Sci-Tech University

[3] Zhao Yan The application of national costume elements in modern clothing design -- Taking Yi nationality in Yongren as an example, $[\mathrm{J}], 2013.10$ middle periods of literature and art

[4] Su Xiaoyan, Liang mountain Yi costume culture and technology. [M] China Textile Press, December 1, 2008. 